\title{
Using Broadcast Wind Observations to Update the Optimal Descent Trajectory in Real-time
}

\author{
Ramon Dalmau* and Xavier Prats ${ }^{\dagger}$ \\ Technical University of Catalonia (UPC), Castelldefels, 08860, Spain \\ Brian Baxley, \\ NASA Langley Research Center (LaRC), Hampton VA, 23681, USA
}

\begin{abstract}
The ability to meet a controlled time of arrival during a continuous descent operation will enable environmentally friendly and fuel efficient descent operations while simultaneously maintaining airport throughput. However, if the wind forecast used to compute the initial trajectory plan is not accurate enough, the guidance system will need to correct time deviations from the plan during the execution of the descent. Previous work proposed an on-board guidance strategy based on model predictive control, which repeatedly updates the trajectory plan in real-time from the current aircraft state and for the remainder of the descent. However, the wind conditions downstream, at altitudes not explored yet, were difficult to predict due to the lack of data. This paper shows the potential benefits of using wind observations, broadcast by nearby aircraft, to reconstruct the wind profile downstream. The wind profile in the trajectory optimization problem is modeled as a spline, which control points are updated to fit the observations before re-planning the trajectory. Results from simulations using realistic wind data show that the performance of model predictive control significantly improves when including up-to-date wind observations, in terms of time and energy errors at the metering fix and fuel consumption.
\end{abstract}

\section{Introduction}

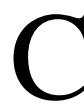
ONTINUOus descent operations (CDOs) with controlled times of arrival (CTAs) at metering fixes could enable more environmentally friendly procedures while simultaneously maintaining (or increasing) airport and airspace throughput. This concept of flight operations assumes a negotiation process between the on-board Flight Management System (FMS) and air traffic controllers (ATC), typically before the descent. This negotiation starts with the on-board computation of the earliest and latest achievable times of arrival at the metering fix, and the subsequent down-link of this information to the ground automation system. Based on this feasible time window and the surrounding traffic, a CTA is

\footnotetext{
*PhD Student and researcher, Department of Physics, Technical University of Catalonia (UPC)

†Serra Húnter Fellow (Associate Professor), Department of Physics, Technical University of Catalonia (UPC)

${ }^{\ddagger}$ Research Engineer, Crew Systems and Aviation Operations Branch, NASA LaRC, AIAA Senior Member.
} 
computed by a ground-based support tool, such as an arrival manager. Then, the incoming CTA is entered on-board as a Required Time of Arrival (RTA) into the FMS, and the on-board trajectory planner computes a new (optimal) trajectory plan starting at the current state, while satisfying the RTA and other operational constraints [1]. These type of flight operations, which goal is to use timing at meter points to more efficiently deliver aircraft to the terminal maneuvering area, are a cornerstone for the queue-management project by the Single European Sky Air traffic management Research (SESAR) program in Europe, and for the Time-Based Flow Management (TBFM) program in the United States.

The RTCA published standards for time of arrival control as $95 \%$ compliance with accuracy levels of $\pm 10 \mathrm{~s}$ for CTAs involving descents subject to the meteorological uncertainty model described in [2]. This model was such that "the average ground speed error due to meteo error (wind and temperature) along a flight plan follows a Gaussian statistical law with mean error of $0 \mathrm{kt}$ and $2 \sigma=10 \mathrm{kt}$ when all wind and temperature met data for the following altitudes are used: FL050, FL100, FL140, FL180, 629 FL240, FL300, FL340, FL390, FL450 and FL530." In this context, several studies investigated the effect of errors in the wind forecast on the performance of CDOs with CTAs [3, 4], and concluded that an accurate knowledge of the actual wind conditions is of utmost importance. For instance, results from a flight test conducted at the Denver International Airport (KDEN) that explored seven different categories of errors in trajectory calculations by the FMS, indicated that approximately two-thirds of the mean time error and nearly all of the standard deviation was due to an incorrect wind forecast [5]. Other flight trials described in Refs. [6 9], also showed the importance of using a high-quality wind forecast during the trajectory planning process to accurately comply with CTAs.

State-of-the-art FMSs compute the optimal descent trajectory plan satisfying applicable CTAs before starting the descent, using the best available forecast of the wind along the route towards the arrival airport. Then, this initial trajectory is frozen and the guidance system uses different strategies to execute it. The initial trajectory plan, however, shows only what can be achieved given perfect knowledge of the actual wind conditions. If the wind forecast used by the FMS does not match the actual wind conditions, the initial trajectory plan is no longer the most optimal, and some operational constraints (including the CTA) may be violated if errors are not actively nullified by the guidance system during the descent. The use of accurate and up-to-date wind data when planning the initial trajectory could potentially reduce the throttle and speed brakes actions commanded by the guidance system while simultaneously improving compliance with CTAs. Moreover, a primary driver of accurate CTA compliance is not only the quality of the wind forecast provided to the FMS, but also the guidance strategy used to execute the trajectory plan [10].

Nonlinear model predictive control (NMPC) is a guidance strategy based on a frequent update of the optimal trajectory plan during the execution of the descent [11]. Previous work [12] showed that NMPC is very robust in terms of correcting energy (speed and altitude) and time deviations, providing at the same time acceptable fuel consumption and noise nuisance figures. Other research [13] has also demonstrated the feasibility of using NMPC to achieve precise spacing between aircraft, the objective of interval management (IM) operations. Generic NMPC algorithms, however, do not always return a feasible solution and/or the computation time is prohibitively long, making this kind of guidance 
an impractical solution in actual operations. An alternative that addresses these short-comings consists of computing fast updates of the active optimal trajectory using the theory of neighboring extremals [14]. Parametric sensitivities are obtained by linearization of the necessary conditions of optimality to rapidly update the optimal trajectory for small perturbations in the parameters of the model. This strategy, known in the literature as sensitivity-based NMPC [15], reduces the execution time while ensuring that operational constraints are satisfied and that the cost is minimized.

In previous work [16], sensitivity-based NMPC was implemented to guide aircraft in CDO subject to CTA. Moreover, the guidance algorithm was enhanced to enable the calculation of the optimal trajectory plan using a wind profile based on the original forecast but progressively updated based on the ownship sensed winds. However, that only corrects the wind profile for current and previous positions, not downstream positions, that is, from current position to destination. The short-coming of using ownship sensed winds is that any time deviation due to an incorrect wind forecast at a downstream waypoint requires a higher and higher change to the aircraft's airspeed as the distance to that point becomes shorter and shorter [10]. Using wind observations from aircraft that have recently crossed downstream waypoints at an altitude similar to that planned by the ownship is expected to improve the accuracy of the ownship trajectory predictions.

References [17-19] proposed wind prediction algorithms that use wind observations broadcast by nearby aircraft to update, on-board and in real-time, the wind profile used to calculate the trajectory plan. Other studies investigating aircraft spacing during IM operations showed that the use of wind predictions generated from observations emitted by aircraft in range within a wind networking concept could reduce the spacing time error if compared with using outdated wind information [20]. In this paper, the NMPC guidance strategy is implemented to guide aircraft during CDOs subject to CTAs. In addition, the proposed guidance system accesses data available in a hypothetical wind networked environment, where aircraft share wind observations, to generate accurate and up-to-date wind predictions on-board and in real-time.

\section{Background}

This paper assesses the benefit of combining a NMPC guidance strategy with updating the wind profile using wind observations broadcasted by nearby aircraft.

\section{A. Nonlinear model predictive control (NMPC)}

NMPC is based on the solution, at each time sample, of an optimal control problem over a future time horizon [11]. The resulting optimal control is applied only until the next time sample, where the optimal control problem is solved again. This process is repeated over and over.

Typical NMPC applications consider a fixed-length time horizon, which advances an interval sample at each trajectory plan re-calculation. Alternatively, when the system has to reach a certain state at a particular time, a shrinking horizon is often preferred. Using this strategy, the length of the horizon is not fixed but decreases by one interval sample 
at each trajectory plan update.

\section{NMPC trajectory optimization}

Many real-life processes, such as the descent of an aircraft, can be divided into several phases (or stages) where the dynamics of the system, the cost function and/or the constraints of the optimization problem might change. Note that, in this paper, the term phase does not refer to the standard phases of a flight (e.g., take-off, climb, cruise), but to the stages of a generic multi-phase optimal control problem [21].

Let the continuous time horizon $\left[t_{I}, t_{F}\right]$ be divided into $P$ time intervals $\left[t_{j}, t_{j+1}\right]$ for $j=0, \ldots, P-1$, where each time interval corresponds to a different phase. Note that $t_{0}=t_{I}$ and $t_{P}=t_{F}$. Then, each time intervals (phase) is discretized into $N_{j}$ equidistant time samples $\tau_{k}, \tau_{k+1}, \ldots, \tau_{k+N_{j}-1}$, where $\tau_{k}=t_{j}, \tau_{k+N_{j}-1}=t_{j+1}$ and $k=\sum_{i<j} N_{i}$, for all $j=0, \ldots, P-1$. The discretization step of the $j^{\text {th }}$ phase (difference between two consecutive time samples) is denoted by $\Delta \tau_{j}$. As a result, the whole time horizon is discretized into $N+1=\sum_{j=0}^{P-1} N_{j}$ time samples $\tau_{0}, \tau_{1}, \ldots, \tau_{N}$.

Let $\mathcal{T}$ be a multi-dimensional set that relates the index of each phase to the indexes of its corresponding time samples. The subset $\mathcal{E} \subseteq \mathcal{T}$ only includes the index corresponding to the last time sample of each phase; and $\mathcal{I}$ is defined as $\mathcal{T} \backslash \mathcal{E}$.

For instance, for a multi-phase optimal control problem with $P=2$ phases and $N+1=8$ time samples equally distributed among them (i.e., $N_{j}=4$ for $j=0,1$ ), these subsets would be particularised as:

$$
\begin{aligned}
\mathcal{T} & =\{(0,0),(0,1),(0,2),(0,3),(1,4),(1,5),(1,6),(1,7)\} \\
\mathcal{E} & =\{(0,3),(1,7)\} \\
\mathcal{I} & =\{(0,0),(0,1),(0,2),(1,4),(1,5),(1,6)\} .
\end{aligned}
$$

The optimal control problem starting at $\tau_{0}$, and minimizing a cost function $J$ (e.g., fuel) in the remaining horizon is:

$$
\begin{array}{ll}
\min _{\substack{\boldsymbol{x}_{k}, k=0, \ldots, N \\
\boldsymbol{u}_{k}, k=0, \ldots, N-1}} J:=\sum_{(j, k) \in \mathcal{E}} \phi_{j}\left(\boldsymbol{x}_{k}, \boldsymbol{d}\right)+\sum_{(j, k) \in \mathcal{I}} \Pi_{j}\left(\boldsymbol{x}_{k}, \boldsymbol{u}_{k}, \boldsymbol{d}, \Delta \tau_{j}\right) \\
\mathrm{s.t} & \boldsymbol{x}_{0}=\boldsymbol{X} \\
& \boldsymbol{x}_{k+1}=\boldsymbol{F}_{j}\left(\boldsymbol{x}_{k}, \boldsymbol{u}_{k}, \boldsymbol{d}, \Delta \tau_{j}\right) ; \forall(j, k) \in \mathcal{I} \\
& \boldsymbol{b}_{j}^{e q}\left(\boldsymbol{x}_{k}, \boldsymbol{u}_{k}, \boldsymbol{d}\right)=0 ; \forall(j, k) \in \mathcal{I} \\
& \boldsymbol{b}_{j}^{i n}\left(\boldsymbol{x}_{k}, \boldsymbol{u}_{k}, \boldsymbol{d}\right) \leq 0 ; \forall(j, k) \in \mathcal{I} \\
& \boldsymbol{\vartheta}_{j}^{e q}\left(\boldsymbol{x}_{k}, \boldsymbol{d}\right)=0 ; \forall(j, k) \in \mathcal{E} \backslash\{(P-1, N) \\
& \boldsymbol{\vartheta}_{j}^{i n}\left(\boldsymbol{x}_{k}, \boldsymbol{d}\right) \leq 0 ; \forall(j, k) \in \mathcal{E} \backslash\{(P-1, N) \\
& \boldsymbol{\psi}\left(\boldsymbol{x}_{N}, \boldsymbol{d}\right)=0 \\
& \boldsymbol{x}_{k}-\boldsymbol{x}_{k+1}=0 ; \forall(j, k) \in \mathcal{E} \backslash\{(P-1, N)\},
\end{array}
$$


where $\boldsymbol{x}_{k} \in \mathfrak{R}^{n_{x}}$ and $\boldsymbol{u}_{k} \in \mathfrak{R}^{n_{u}}$ are the state and control vectors discretized at $\tau_{k}$, respectively; $\boldsymbol{d} \in \mathfrak{R}^{n_{d}}$ is the vector of fixed parameters of the model; $\boldsymbol{b}_{j}^{e q}: \mathfrak{R}^{n_{x}} \times \mathfrak{R}^{n_{u}} \times \mathfrak{R}^{n_{d}} \rightarrow \mathfrak{R}^{n_{\varphi_{j}}}$ and $\boldsymbol{b}_{j}^{\text {in }}: \mathfrak{R}^{n_{x}} \times \mathfrak{R}^{n_{u}} \times \mathfrak{R}^{n_{d}} \rightarrow \mathfrak{R}^{n_{b_{j}}}$ are the algebraic and path constraints, respectively, of the $j^{\text {th }}$ phase; $\boldsymbol{\vartheta}_{j}^{e q}: \mathfrak{R}^{n_{x}} \times \mathfrak{R}^{n_{d}} \rightarrow \mathfrak{R}^{n_{\vartheta_{j}^{e q}}^{e q}}$ and $\boldsymbol{\vartheta}_{j}^{\text {in }}: \mathfrak{R}^{n_{x}} \times \mathfrak{R}^{n_{d}} \rightarrow \mathfrak{R}^{n_{\vartheta_{j}^{i n}}}$ represent applicable equality and inequality interior-point constraints, respectively, applied at the last time of the $j^{\text {th }}$ phase. These constraints will be mainly used to model speed and/or altitude constraints at the different waypoints of the route. The continuity of the state vector across consecutive phases is ensured by another type of constraints: the link constraints (last term in Eq. (1)). Furthermore, $\psi: \mathfrak{R}^{n_{x}} \times \mathfrak{R}^{n_{d}} \rightarrow \mathfrak{R}^{n_{\psi}}$ are the terminal constraints; and $\Pi_{j}: \mathfrak{R}^{n_{x}} \times \mathfrak{R}^{n_{u}} \times \mathfrak{R}^{n_{d}} \times \mathfrak{R} \rightarrow \mathfrak{R}$ and $\boldsymbol{F}_{j}: \mathfrak{R}^{n_{x}} \times \mathfrak{R}^{n_{u}} \times \mathfrak{R}^{n_{d}} \times \mathfrak{R} \rightarrow \mathfrak{R}^{n_{x}}$ are the quadrature and states evolution functions for the $j^{\text {th }}$ phase, respectively, which are the result of integrating the continuous running cost $\left(\pi_{j}\right)$ and the system ordinary differential equations (ODEs) describing the dynamics of the system $\left(\boldsymbol{f}_{j}\right)$ during an interval of duration $\Delta \tau$ using the discretized states and controls. For instance, using the simple Euler method, these functions would be:

$$
\begin{aligned}
& \boldsymbol{F}_{j}\left(\boldsymbol{x}_{k}, \boldsymbol{u}_{k}, \boldsymbol{d}, \Delta \tau_{j}\right)=\boldsymbol{x}_{k}+\boldsymbol{f}_{j}\left(\boldsymbol{x}_{k}, \boldsymbol{u}_{k}, \boldsymbol{d}\right) \Delta \tau_{j} \\
& \Pi_{j}\left(\boldsymbol{x}_{k}, \boldsymbol{u}_{k}, \boldsymbol{d}, \Delta \tau_{j}\right)=\pi_{j}\left(\boldsymbol{x}_{k}, \boldsymbol{u}_{k}, \boldsymbol{d}\right) \Delta \tau_{j} ; \forall(j, k) \in \mathcal{I}
\end{aligned}
$$

Note that the discretization step of each individual phase could be considered either a known parameter or variable to be optimized, depending on the context. For instance, if the duration of the whole time horizon were fixed to a certain parameter, say a CTA, but the duration of each phase were flexible, $\Delta \tau_{j}$ for $j=0, \ldots, P-1$ would become additional decision variables subject to the following constraint:

$$
\sum_{j=0}^{P-1}\left(N_{j}-1\right) \Delta \tau_{j}-\mathrm{CTA}=0
$$

which would be appended to Eq. (1).

It should be noted that the vector of fixed parameters of the model $(\boldsymbol{d})$, however, is not part of the decision variables and, consequently, must be chosen by the user and remains constant during the whole optimization process. This vector could include, for instance, parameters describing the weather forecast.

The optimal trajectory plan can be computed by formulating the discrete-time optimal control problem Eq. (1) as the following parametric nonlinear programming (NLP) optimization problem:

$$
\begin{array}{ll}
\min _{z} & f(z, \boldsymbol{p}) \\
\text { s.t } & \boldsymbol{h}(\boldsymbol{z}, \boldsymbol{p})=0 \\
& \boldsymbol{g}(\boldsymbol{z}, \boldsymbol{p}) \leq 0,
\end{array}
$$


where $z \in \mathfrak{R}^{n_{z}}$ is the vector of primal variables; $\boldsymbol{h}: \mathfrak{R}^{n_{z}} \times \mathfrak{R}^{n_{p}} \rightarrow \mathfrak{R}^{n_{h}}$ and $\boldsymbol{g}: \mathfrak{R}^{n_{z}} \times \mathfrak{R}^{n_{p}} \rightarrow \mathfrak{R}^{n_{g}}$ are the vectors of equality and inequality constraints, respectively; and $\boldsymbol{p} \in \mathfrak{R}^{n_{p}}$ is the vector of (fixed) parameters of the NLP optimization problem. In Eq. (4), $f$ is the cost function of the original optimal control problem Eq. (1) evaluated at the primal variables and fixed NLP parameters, i.e., $f(z, p)=J(z, p)$. In this paper, the vector of fixed NLP parameters is composed of the initial conditions $(\boldsymbol{X})$ and the parameters of the model, i.e., $\boldsymbol{p}=[\boldsymbol{X}, \boldsymbol{d}]^{T}$. Moreover, the following notation has been used:

$$
\begin{aligned}
z & :=\left[z_{0}, z_{1}, \ldots, z_{N}\right]^{T} \\
\boldsymbol{h}(z, \boldsymbol{p}) & :=\left[\boldsymbol{h}_{0}(z, \boldsymbol{p}), \boldsymbol{h}_{1}(z, \boldsymbol{p}), \ldots, \boldsymbol{h}_{N}(z, \boldsymbol{p})\right]^{T} \\
\boldsymbol{g}(\boldsymbol{z}, \boldsymbol{p}): & :=\left[\boldsymbol{g}_{0}(z, \boldsymbol{p}), \boldsymbol{g}_{1}(z, \boldsymbol{p}), \ldots, \boldsymbol{g}_{N}(z, \boldsymbol{p})\right]^{T}
\end{aligned}
$$

where $(\cdot)^{T}$ represents the transpose of $(\cdot)$ and

$$
\begin{aligned}
& \boldsymbol{z}_{k}:= \begin{cases}{\left[\boldsymbol{u}_{k}, \boldsymbol{x}_{k}\right]^{T}} & \text { if } k \neq N \\
\boldsymbol{x}_{k} & \text { if } k=N\end{cases} \\
& \boldsymbol{h}_{k}:= \begin{cases}{\left[\boldsymbol{x}_{k+1}-\boldsymbol{F}_{j, k}, \boldsymbol{b}_{j, k}^{e q}\right]^{T}} & \text { if }(j, k) \in \mathcal{I} \\
{\left[\boldsymbol{\vartheta}_{j, k}^{e q}, \boldsymbol{x}_{k}-\boldsymbol{x}_{k+1}\right]^{T}} & \text { if }(j, k) \in \mathcal{E} \backslash\{P-1, N\} \\
\boldsymbol{\psi}\left(\boldsymbol{x}_{k}, \boldsymbol{d}\right) & \text { if } k=N\end{cases} \\
& \boldsymbol{g}_{k}:= \begin{cases}\boldsymbol{b}_{j, k}^{i n} & \text { if }(j, k) \in \mathcal{I} \\
\boldsymbol{\vartheta}_{j, k}^{i n} & \text { if }(j, k) \in \mathcal{E} \backslash\{P-1, N\} .\end{cases}
\end{aligned}
$$

According to Eq. (6), $z_{k}$ includes both discretized states and controls at the time sample $\tau_{k}$. Similarly, $\boldsymbol{g}_{k}$ and $\boldsymbol{h}_{k}$ include the inequality and equality constraints applied at $\tau_{k}$.

Furthermore, in order to reduce the number of variables and constraints, the constraint that determines the initial condition of the optimal control problem to the current state of the system is eliminated by substituting the variables $\boldsymbol{x}_{0}$ for the initial conditions $\boldsymbol{X}$ in the whole NLP optimization problem. This makes the constraint $\boldsymbol{x}_{0}=\boldsymbol{X}$ of Eq. (1) redundant, allowing to remove the variable $\boldsymbol{x}_{0}$ from the calculations.

Let $\mathcal{P}_{N}$ be the NLP algorithm that provides the optimal primal-dual solution as a function of $\boldsymbol{p}$ for the next $N$ samples:

$$
\left(z^{*}, \lambda^{*}, \mu^{*}\right) \leftarrow \mathcal{P}_{N}(\boldsymbol{p})
$$

where $\lambda \in \mathfrak{R}^{n_{g}}$ and $\boldsymbol{\mu} \in \mathfrak{R}^{n_{h}}$ are the Lagrange multipliers (dual variables) paired up with the constraints $\boldsymbol{g}$ and $\boldsymbol{h}$, 
respectively. A more technical description of the conditions necessary for optimality is found in Ref. [16].

When using the shrinking horizon NMPC, the optimal control problem is solved at each $\tau_{i}, i=0, \ldots, N-1$ in order to obtain the optimal trajectory starting at $\tau_{i}$ and extending to $\tau_{N}$. Let $\mathcal{P}_{N-i}$ be the NLP algorithm that provides the optimal primal-dual solution as a function of $\boldsymbol{p}$, but starting at a generic $\tau_{i}$ instead of $\tau_{0}$ :

$$
\left(z_{i:}^{*}, \lambda_{i:}^{*}, \boldsymbol{\mu}_{i:}^{*}\right) \leftarrow \mathcal{P}_{N-i}(\boldsymbol{p})
$$

where $(\cdot)_{i}$ indicates the elements of $(\cdot)$ corresponding to time samples from $\tau_{i}$ to the end of the time horizon $\left(\tau_{N}\right)$.

\section{NMPC guidance strategy}

In an ideal case, problem $\mathcal{P}_{N-i}$ is solved at each $\tau_{i}$, as soon as the parameter vector $\boldsymbol{p}$ is measured or estimated. Then, the resulting optimal control $\boldsymbol{u}_{i}^{*}$ is applied without delay until $\tau_{i+1}$, where the process is repeated. However, for achieving optimal performance and good stability properties, problem $\mathcal{P}_{N-i}$ needs to be solved instantaneously. We refer to this hypothetical case as the ideal NMPC (INMPC). Algorithm 1 details its main steps.

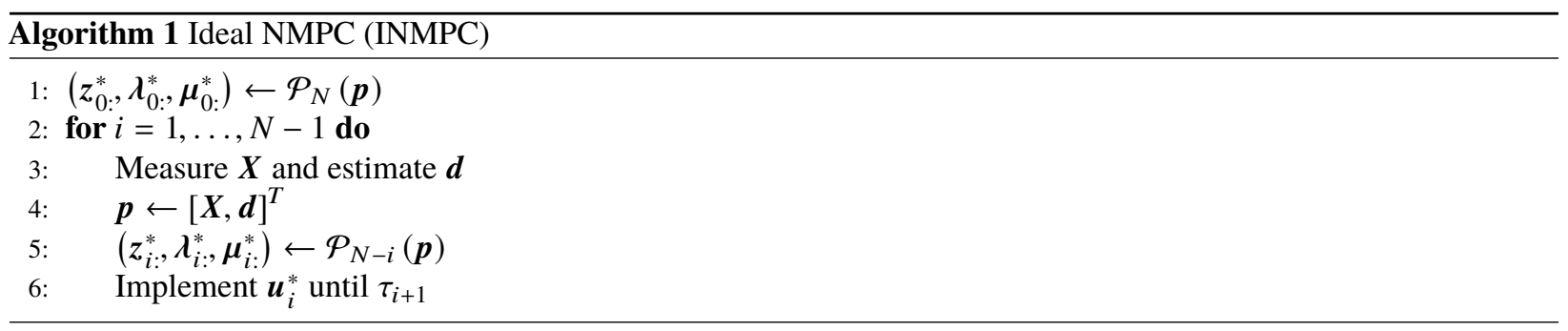

In practical applications $\mathcal{P}_{N-i}$ may be computationally expensive to solve. This implies that the control $\boldsymbol{u}_{i}^{*}$ cannot be applied just after $\boldsymbol{p}$ is measured or estimated, but only after $\mathcal{P}_{N-i}$ is solved. The delay in calculating the new solution may lead to sub-optimal trajectories, failure to meet constraints, or in some instances instabilities of the solution [22]. This motivates the introduction of sensitivity-based methods, which rapidly update the optimal descent trajectory plan by using parametric sensitivities instead of solving a rigorous NLP optimization problem at each time sample.

Previous work [16] showed that the performance of sensitivity-based methods are similar to those of the INMPC, with the sensitivity-based methods being simpler, faster and more robust. Since the performance was similar, it is expected that the results from the addition of wind networking to the INMPC guidance strategy described in this paper should also apply to the sensitivity-based strategy.

\section{B. Wind sources and models}

Section II.B.1 describes the sources of weather data and the wind models used by state-of-the-art FMSs to generate the trajectory plan. Section II.B.2 presents basic concepts of wind networking and lists some relevant works on this topic. 


\section{Conventional wind sources and models}

Currently, the flight crew receives the wind forecast from the flight dispatcher and either enters that information manually into the FMS or automatically via a data communication system.

The typical FMS allows to store the forecast wind direction and speed at each departure and arrival waypoint, and for up to five altitudes/flight levels at en-route waypoint. Operationally, the five altitudes are usually above, at, and below the aircraft's expected altitude, allowing the FMS to interpolate between the altitudes. When required, the FMS can also extrapolate beyond those altitudes or limits to perform trajectory predictions.

The wind data entered to the FMS are primarily based on wind charts from numerical weather prediction models. Nowadays, the observations required to initialize these models are mainly gathered from radiosondes and aircraft equipped with AMDAR (aircraft meteorological data relay). However, the spatial distribution of the radiosondes, which are launched only two to four times a day, is too coarse, and wind observations gathered through AMDAR are not sufficient because not all aircraft in operation are equipped with that system [23].

Due to the relatively low spatio-temporal resolution of the data used to initialize numerical weather prediction models and the computational burden of running a prediction, typical weather forecast products generate wind predictions only three to eight times per day, and are valid until $+6,+12$ or +24 hours beyond their issued time. Consequently, the wind forecast for the descent is not tailored for the current as-amended flight plan, and could be several hours old by the time the top of descent (TOD) is reached.

Most flight planning suppliers currently use the world area forecast model that is produced to an ICAO specification by meteorological providers. Some flight plan suppliers also generate their own wind forecasts at a finer resolution, but do not provide a higher update rate due to the time required to up-link the data to the FMS using the available bandwidth [24].

\section{Wind networking}

Aircraft could be used as a network of airborne sensors emitting the sensed wind to ground systems [25] and/or to nearby aircraft [26] to provide accurate, high-resolution and up-to-date wind data replacing or complementing the forecasts obtained from numerical weather prediction models. At present, however, aircraft rarely broadcast this valuable information.

Fortunately, the wind vector can be indirectly inferred from standard surveillance data already in place. Several works already proposed to use wind observations derived from surveillance data to provide enhanced wind predictions for the air traffic management (ATM) community. Most wind estimation methods found in the literature rely on the fact that the wind vector is the difference between the ground speed vector and the true airspeed (TAS) vector.

Early in the 1980s, Ref. [25] was the first to propose a method to estimate the wind vector from ground-based (radar) surveillance observations. The ground speed vectors for every scan of each aircraft track were obtained by taking the 
difference between the sequential radar positions of aircraft and then dividing by the scan interval. Then, the ground speed vectors from multiple aircraft were used to infer the wind vector by means of Bayesian estimation techniques, assuming constant wind speed and aircraft airspeed during turns.

Aircraft equipped with Automatic Surveillance-Broadcast (ADS-B) autonomously transmit surveillance data including not only aircraft position but also ground speed vector. The wide availability of ADS-B receivers at a relatively low cost and the growing amount of aircraft equipped with this surveillance system make ADS-B an attractive source of data for many ATM applications. Similarly to Refs. [25] and [27], Ref. [26] proposed a method to estimate the wind vector from ground speed vector observations. In this case, however, the ground speed vector was directly obtained from the ADS-B surveillance messages. The method was based on a modified extended Kalman filter that estimates the wind recursively from an aircraft in a turn. In addition, a new method to estimate wind using data from multiple aircraft was also proposed.

Mode-S messages emitted by the aircraft surveillance system as a response to a secondary surveillance radar interrogation includes information about the ground speed and TAS vector, from which the wind vector can be directly inferred. The use of Mode-S data for wind networking applications was investigated in [28]. Recently, Ref. [29] applied geostatistical techniques to generate a four-dimensional wind model for the terminal maneuvering area; and Ref. [30] investigated a novel and relatively fast gas particle model that estimates the wind field in real-time from ADS-B and Mode-S messages.

In summary, the capability to derive the sensed wind data from aircraft transmitting ADS-B and Mode-S messages will provide the FMS more accurate information from which to calculate the optimal trajectory. Nearby aircraft, in particular those on the same trajectory, broadcast data that can be used to update and improve the wind profile used by the FMS to optimize the trajectory while meeting constraints and CTAs. Last but not least, it is important to remark that updates to the ADS-B message standard (currently being worked in RTCA Special Committee 186) will directly include the broadcast of weather information, such as wind speed and direction, and temperature.

\section{NMPC guidance strategy for a time-constrained CDO}

In this Section the generic optimal control Eq. (1) problem is particularized for an aircraft already in descent on a CDO that is subject to a time constraint at a single metering fix. Then, an algorithm to estimate the wind profile from observations is proposed.

\section{A. Optimal control problem formulation}

The state vector $\boldsymbol{x}=[t, v, h]^{T}$ is composed of time, TAS, and altitude; the control vector $\boldsymbol{u}=[\gamma, T, \beta]^{T}$ is composed of the aerodynamic flight path angle, engine thrust, and speed brakes deflection. The flight path angle is the control that is used by the aircraft to modulate energy (i.e., exchange potential energy for kinetic energy and vice-versa), whereas 
thrust and speed brakes are used to add and remove energy.

Different from typical approaches, the independent variable is the distance to go $(s)$ and not the time. The selection of $s$ as the independent variable is driven by the fact that during an ideal CDO, with no intervention from the ATC except for the assignment of the CTA, the aircraft will follow a defined route and the remaining distance to go will be known. In addition, this formulation replicates how constraints are defined in the current operational environment, thereby enabling more precise modeling of the operational constraints at the different waypoints and legs.

The dynamics of $\boldsymbol{x}$ are expressed by the following ODEs, considering a point-mass representation of the aircraft reduced to a gamma-command model, where vertical equilibrium is assumed and the cross and vertical winds are assumed negligible:

$$
\boldsymbol{f}_{j}=\frac{\mathrm{d} \boldsymbol{x}}{\mathrm{d} s}=\left[\begin{array}{c}
1 \\
\frac{T-D(v, h, \beta)}{m}-g \sin \gamma \\
v \sin \gamma
\end{array}\right] \frac{1}{v \cos \gamma+w(h)},
$$

where $D: \mathbb{R}^{n_{x} \times n_{u}} \rightarrow \mathbb{R}$ is the aerodynamic drag; $g$ is the gravity acceleration; $m$ is the mass, which is assumed to be constant since the fuel consumption during a descent is a small fraction of the total mass [31]; and the longitudinal component of the wind $w: \mathbb{R} \rightarrow \mathbb{R}$ is modeled by a smoothing spline [32]:

$$
w(h)=\sum_{i=1}^{n_{c}} c_{i} B_{i}(h)
$$

where $B_{i}, i=1, \ldots, n_{c}$, are the B-spline basis functions and $\boldsymbol{c}=\left[c_{1}, \ldots, c_{n_{c}}\right]$ are control points of the smoothing spline. It should be noted that the longitudinal wind has been modeled as a function of the altitude only, as done in similar works [18].

The goal of the NMPC guidance is to minimize a weighted sum of the fuel consumption and speed brakes use (which leads to airframe noise) for the remaining descent. Thus, the running cost is:

$$
\pi_{j}=\frac{q(v, h, T)+K_{\beta} \beta}{(v \cos \gamma+w(h))}
$$

where $q: \mathbb{R}^{n_{x} \times n_{u}} \rightarrow \mathbb{R}$ is the fuel flow and $K_{\beta}$ a parameter that determines how much the use of speed brakes is penalized.

Furthermore, generic phase-independent path constraints on the controls ensure that the maximum and minimum descent gradients, thrust and speed brakes are not exceeded throughout the descent: 


$$
\boldsymbol{b}_{j}^{i n}=\left[\begin{array}{c}
\gamma \\
\gamma_{\min }-\gamma \\
T_{\min }-T \\
T-T_{\max } \\
-\beta \\
\beta-1
\end{array}\right],
$$

where $\gamma_{\text {min }}$ is the maximum descent gradient; $T_{\text {min }}: \mathbb{R}^{n_{x}} \rightarrow \mathbb{R}$ and $T_{\text {max }}: \mathbb{R}^{n_{x}} \rightarrow \mathbb{R}$ are the idle and maximum thrust, respectively; $\beta=0$ and $\beta=1$ indicate that speed brakes are retracted and fully extended, respectively.

Different alternatives can be used to model the aircraft performance functions $T_{\min }, T_{\max }, D$ and $q$ and their respective parameters. In this paper, the EUROCONTROL's base of aircraft data (BADA) v4 model has been adopted [33]. However, BADA v4 does not include a model for the effects of the speed brakes on the drag coefficient $\left(C_{D}\right)$. As a workaround, in this paper the contribution of the speed brakes is modeled as an extra linear term $C_{D_{\beta}} \beta$ in the generic BADA v4 drag coefficient model, where $C_{D_{\beta}}$ is a coefficient representing the increase in drag coefficient for unit of speed brakes deflection.

It should be noted that operational constraints in aviation are usually given in terms of the Mach number or calibrated airspeed (CAS), which can be expressed as function of the state vector. On the one hand, the Mach number $M: \mathbb{R}^{n_{x}} \rightarrow \mathbb{R}$ is defined as the ratio between the true airspeed of the aircraft and the speed of sound, which depends on the temperature of the air:

$$
M(h, v)=\frac{v}{\sqrt{\gamma_{a} R \theta(h), \tau_{S S L}}},
$$

where $R$ is the perfect gases constant, $\gamma_{a}$ is the specific heat of the air, and the function $\theta(h): \mathfrak{R} \rightarrow \mathfrak{R}$ is the temperature of the air normalised with respect to the temperature at standard sea-level conditions $\left(\tau_{S S L}=288.15 \mathrm{~K}\right)$.

On the other hand, the CAS is defined as:

$$
v_{C A S}(h, v)=\sqrt{\frac{2 \gamma_{a}}{\gamma_{a}-1} \frac{p_{S S L}}{\rho_{S S L}}\left((\delta(h) B(h, v)+1)^{\frac{\gamma a-1}{\gamma a}}-1\right)},
$$

where $\sigma(h): \mathfrak{R} \rightarrow \mathfrak{R}$ and $\delta(h): \mathfrak{R} \rightarrow \mathfrak{R}$ are, respectively, the density and pressure of the air normalised with respect to standard sea-level conditions $\left(\rho_{S S L}=1.225 \mathrm{~kg} \mathrm{~m}^{-1}\right.$ and $\left.p_{S S L}=101.325 \mathrm{kPa}\right)$, and:

$$
B(h, v):=\left(\frac{1}{2} \frac{\gamma_{a}-1}{\gamma_{a}} v^{2}\left(\frac{\sigma(h) \rho_{S S L}}{\delta(h) p_{S S L}}\right)+1\right)^{\frac{\gamma_{a}}{\gamma_{a}-1}}-1 .
$$


Generally speaking, $\theta, \delta$ and $\sigma$ are functions of both altitude and distance to go, which can be directly mapped to the aircraft's position in the earth. In practise, however, most common atmospheric models, such as the International Standard Atmosphere (ISA) used in this paper, assume that these variables are only function of the altitude.

The descent is divided into $P$ phases, defined between two consecutive waypoints of the lateral route with associated operational speed and/or altitude constraints. In each phase, the different operational constraints that may apply can be modeled in the form of path, algebraic and/or interior-point constraints (see Eq. (1)). The exact number of phases and associated operational constraints depend on the particular procedure being investigated. The phases for the procedure modelled in the experiment of this paper will be presented in Section IV.A

Finally, terminal constraints fix the state vector at the metering fix are defined as:

$$
\psi=\left[\begin{array}{c}
t-\mathrm{CTA} \\
v_{C A S}(h, v)-v_{C A S_{F}} \\
h-h_{F}
\end{array}\right],
$$

where $v_{C A S_{F}}$ and $h_{F}$ are the CAS and altitude at the metering fix.

In the model proposed in this paper, the vector of model parameters includes the control points of the spline approximating the longitudinal wind and the CTA, i.e., $\boldsymbol{d}=[\boldsymbol{c}, \mathrm{CTA}]^{T}$. This definition allows the optimal trajectory to be updated whenever an improved wind forecast is available or the CTA is tactically modified by ATC.

\section{B. Update of wind profile}

Remember from previous Section that the mathematical function that has been used to represent the wind profile is called spline. A spline is a piece-wise function that has to main parameters: the list of knots and the list of control points. The places where the pieces meet are the knots. One could enforce the spline to pass through all data points, but this typically results in noisy derivatives which are not numerically-friendly from the optimization point of view [21]. A well-known technique for eliminating these oscillations is to approximate the data points rather than interpolating them. This can be achieved by using smoothing splines, which minimize the curvature of the spline whilst ensuring sufficient accuracy through the smoothing factor. In this paper, the vector of knots is fixed to a set of flight levels, and the control points are modified to approximate the wind observations.

Given a set $O$ composed by $n_{o}$ wind observations with associated time stamps $\left(\hat{h}_{k}, \hat{w}_{k}, \tau_{k}\right), k=1, \ldots, n_{o}$, and a vector of fixed knots, the optimal location of the control points $c_{k}, k=1, \ldots, n_{c}$, that minimize the curvature of the smoothing spline while bounding the approximation error is obtained by solving the following weighted least-squares fitting problem: 


$$
\begin{array}{ll}
\min _{c_{k}, k=1, \ldots, n_{c}} & \int w^{\prime \prime}(h)^{2} \mathrm{~d} h \\
\text { s.t } & \sum_{k=1}^{n_{o}} \omega_{k}\left(w\left(\hat{h}_{k}\right)-\hat{w}_{k}\right)^{2} \leq \varepsilon
\end{array}
$$

where $\varepsilon$ specifies the trade-off between smoothness and accuracy of the approximation. The weights associated with the observations can be defined in many different ways. In this paper, the weights are updated at each time sample $\tau_{i}$

according to $\omega_{k}=\Lambda^{\tau_{k}-\tau_{i}}$, where $\tau_{k}$ is the time sample when the observation at $\hat{h}_{k}$ was obtained, independently of the source of information and its geographical location. As such, the forgetting factor $\Lambda \in[0,1]$ weights the more recent measurements so that old observations are discounted at an exponential rate.

\section{Experiment setup}

The CDO procedure selected for the experiment is currently in use at KDEN. From this original procedure, only a subset of altitude and speed constraints at four waypoints were considered in the model. The phases and associated constraints of this procedure model will be presented in Section IV.A. The performance of the proposed aircraft guidance concept for the resulting procedure model was assessed for 10 case studies. For each case study, the initial trajectory plan was computed with a different weather forecast obtained from historical data. Then, the execution of the descent was performed in a flight simulator that included the actual weather for the associated forecast. This approach allowed to generate realistic differences between the wind profile forecast used in the computation of the trajectory plan and the actual wind found during the descent. The 10 case studies are listed in Section IV.B. Finally, each one of the 10 case studies was executed assuming three different wind observation rates during the descent (i.e., expected number of wind observations obtained per time step right before updating the wind profile forecast), assuming a static wind forecast (i.e., not updating the wind forecast at all), and the open-loop execution of the initial trajectory plan.

\section{A. Arrival route}

The BOSSS TWO standard arrival procedure at KDEN was selected as the starting point to define the lateral route and the vertical profile used in this paper. The metering fix where CTAs were assigned by an hypothetical ATC during the simulations was the final approach point (DYMON). A more detailed view of this procedure can be found in [16].

From all the waypoints of the route, only the altitude and speed constraints at QUAIL, BOSSS, CHAPP and DYMON were modeled. In order to accomplish that, the descent was divided in $P=4$ different phases, with associated phase-dependent path, algebraic and/or interior-point constraints. It should be noted, however, that the fact of modeling few constraints of the real procedure is not a shortcoming nor a limitation of the model. The model proposed in this paper can handle an unlimited number of phases and associated constraints, yet few constraints have been selected aiming to represent a futuristic and less restricted procedure facilitating CDOs, as well as to ease the interpretation 
of the results. Generally speaking, the complexity of the numerical optimization problem and, consequently, the execution time, increases with the number of phases and constraints. In some specific situations with very non-linear constraints and/or cost function, the convergence of the algorithm might be also compromised. Unfortunately, it is not straightforward to establish a comprehensive relationship between the number of phases and constraints and the execution time. This is one of the well-known limitations of NLP optimization algorithms for real-life applications, since relatively small changes in the model could lead to completely different convergence behaviours.

Table 1 wraps up the different phases and constraints, and Fig. 1 illustrates the KDEN procedure model.

Table 1 Phases and associated constraints for the BOSSS TWO

\begin{tabular}{|c|c|c|c|c|}
\hline Phase & $\boldsymbol{b}_{j}^{i n}$ & $\varphi_{j}^{e q}$ & $\boldsymbol{\vartheta}_{j}^{i n}$ & $\boldsymbol{\vartheta}_{j}^{e q}$ \\
\hline 0 & {$\left[\begin{array}{c}M-\mathrm{MMO} \\
v_{C A S}-\mathrm{VMO} \\
250 \mathrm{kt}-v_{C A S}\end{array}\right]$} & - & {$\left[\begin{array}{l}\text { FL170 }-h \\
h-\text { FL190 }\end{array}\right]$} & {$\left[v_{C A S}-250 \mathrm{kt}\right]$} \\
\hline 1 & {$\left[\begin{array}{l}v_{C A S}-250 \mathrm{kt} \\
210 \mathrm{kt}-v_{C A S}\end{array}\right]$} & - & - & {$\left[\begin{array}{l}v_{C A S}-210 \mathrm{kt} \\
h-12,000 \mathrm{ft}\end{array}\right]$} \\
\hline 2 & {$\left[\begin{array}{c}v_{C A S}-210 \mathrm{kt} \\
\mathrm{GD}-v_{C A S}\end{array}\right]$} & {$[\dot{h}]$} & - & - \\
\hline 3 & {$\left[\begin{array}{c}v_{C A S}-210 \mathrm{kt} \\
\mathrm{GD}-v_{C A S}\end{array}\right]$} & - & - & \\
\hline
\end{tabular}

In Table 1. MMO and VMO are the maximum operative Mach and CAS, respectively; and GD is the green dot speed In this experiment, the maximum descent gradient was set to $-7^{\circ}$. In addition, the values for VMO and MMO were obtained from the BADA v4 global parameters file. The terminal constraints of the generic model (see Eq. (16) were set at the waypoint DYMON, such that $h_{F}=7,000 \mathrm{ft}, v_{C A S_{F}}=\mathrm{GD}=200 \mathrm{kt}$.

Remember that, as described in Section II.A $b_{j}^{i n}$ and $\varphi_{j}^{e q}$ are the inequality and equality algebraic path constraints, respectively, applying all along the $j^{\text {th }}$ phase. Analogously, $\boldsymbol{\vartheta}_{j}^{i n}$ and $\boldsymbol{\vartheta}_{j}^{e q}$ represent inequality and equality interior-point constraints, respectively, enforced at the end of the $j^{\text {th }}$ phase.

\section{B. Case studies}

Accurate wind data were obtained from the rapid refresh (RAP) forecast/analysis system of the National Oceanic and Atmospheric Administration (NOAA). This system generates numerical weather forecasts hourly for look-ahead times up to +18 hours in a $13 \mathrm{~km}$ resolution grid covering North America and for 50 vertical levels extending up to 10 hPa. Slightly different, RAP analyses, which reproduce the actual weather conditions, are generated hourly by using observations gathered from commercial aircraft, balloons, radars and satellites.

\footnotetext{
*For the Airbus A320, the green dot speed is the minimum operating speed in managed mode and clean configuration, being approximately the best lift-to-drag ratio speed
} 


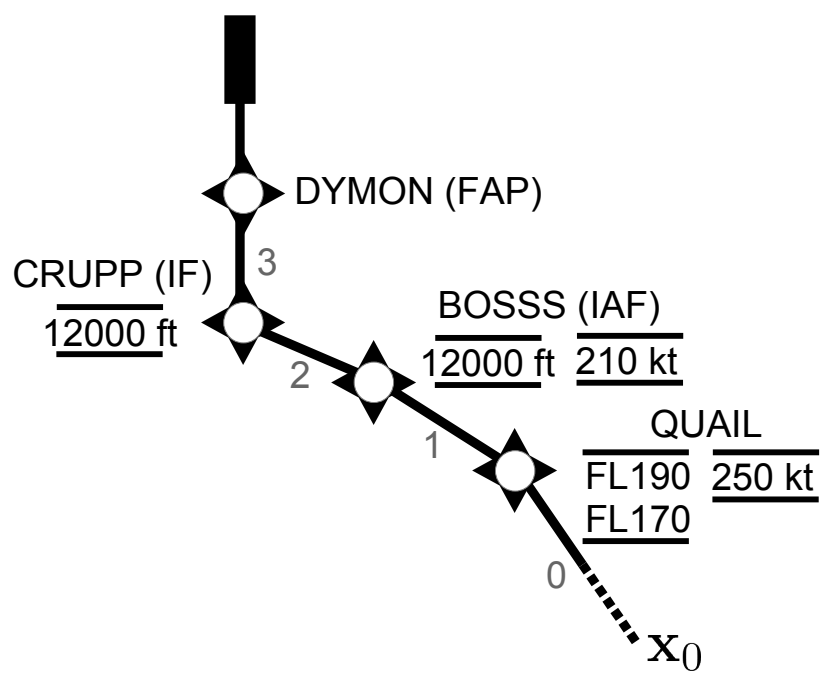

Fig. 1 Phases and associated constraints for the BOSSS TWO

Historical RAP wind forecasts for look-ahead times of +3 and +6 hours during one year (from June 2017 to June 2018) were compared with actual wind data as reported by the corresponding RAP analysis. From these data, the root-mean-square error (RMSE) of each wind forecast over the region of interests (around Denver) up to FL400 was computed.

For each one of the look-ahead times considered in this paper, the 5 RAP forecasts with the highest RMSE were selected. Table 2 lists the case studies selected for the experiment.

Table 2 Case studies

\begin{tabular}{cccc}
\hline Case Study & Look-ahead time & Forecast generation & RMSE $[\mathrm{kt}]$ \\
\hline 00 & & $18-04-2100: 00$ & 18.6 \\
01 & & $18-05-2900: 00$ & 13.3 \\
02 & +3 & $18-04-17$ 18:00 & 11.4 \\
03 & & $17-06-1018: 00$ & 9.9 \\
04 & & $18-05-1418: 00$ & 9.5 \\
\hline 05 & & $18-04-3018: 00$ & 14.1 \\
06 & & $17-06-1218: 00$ & 12.9 \\
07 & +6 & $17-12-0406: 00$ & 12.8 \\
08 & & $17-11-1618: 00$ & 12.2 \\
09 & & $17-09-1918: 00$ & 12.1 \\
\hline
\end{tabular}

\section{Generic simulation workflow}

The experiment simulated an Airbus A320-214 cruising at FL360 and Mach 0.78. Well before starting the descent,

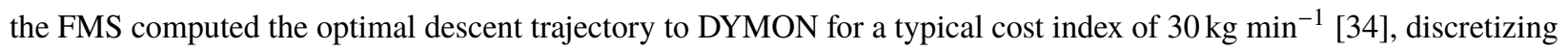


the continuous optimal control problem into $N=60$ time samples. The initial plan was computed considering a smoothing spline for the longitudinal wind profile that approximated the RAP wind forecast data. As a result of this optimization process, the best distance to go from the top of descent, $s_{\mathrm{TOD}}$, and the optimal time of arrival at DYMON were obtained. In addition, the difference between the latest and earliest time of arrival that could be achieved from $s_{\text {TOD }}$ to DYMON without requiring to add or remove energy throughout the descent was also computed.

Note that the total energy of the aircraft is defined as the sum of potential and kinetic energy:

$$
E(h, v)=m g h+\frac{1}{2} m v^{2}
$$

By differentiating Eq. (18) and combining with Eq. (9), the energy rate of the aircraft can be expressed as a function of the thrust and speed-brakes deflection angle:

$$
\dot{E}(h, v, T, \beta)=v(T-D(h, v, \beta))
$$

For this reason, the difference between the latest and earliest time of arrival that could be achieved without requiring neither additional thrust nor speed brakes use throughout the descent is known as the energy-neutral time window. Previous works showed that energy-neutral time windows up to 4 minutes could be obtained for certain flight conditions [29]. The sensitivity of the energy-neutral time window to the state and mass of the aircraft, position of the metering fix along the route, and longitudinal wind speed during the descent was also quantified in [29].

Let us also define the pseudo-specific energy $\left(E_{S}\right)$ as the total energy of the aircraft divided by the aircraft weight:

$$
E_{S}(h, v)=\frac{E(h, v)}{m g}=h+\frac{1}{2} \frac{v^{2}}{g}
$$

The pseudo-specific energy error at the metering fix will be used to assess the performance of the approach proposed in this paper. Note that, by definition, the units of the pseudo-specific energy are ft. Furthermore, the "pseudo" prefix comes from the fact that the components within the state vector are defined with respect to two different reference frames (ground and air mass), which are both used in the same equation to calculate $E_{S}$.

After computing the energy-neutral time window at DYMON, the FMS set a random CTA within the energy-neutral time window as a terminal constraint for the time state in Eq. 16 (assuming an uniform probability distribution between earliest and latest times of arrival), and calculated the optimal descent trajectory from the current state to DYMON by solving $\mathcal{P}_{N}$. All simulations started with the aircraft located at the TOD, ready to start the execution of the optimal descent trajectory using the INMPC guidance strategy (see Algorithm 1). Note that the position of the TOD was computed by using the initial wind forecast from RAP (without incorporating measured winds) at the start of the simulation, and fixed afterwards. Consequently, it may be different for each case study, but does not depend neither on 
the guidance strategy (NMPC or open-loop) nor on the rate of wind observations.

At each time sample, the wind measured by the aircraft sensors at the current altitude and the wind observations received during the last time interval from aircraft in the neighborhood were appended to $O$. Then, the control points of the spline approximating the wind profile were updated by solving Eq. (17). In this paper, the number of wind observations received from aircraft in the neighborhood during a time interval was modeled as a Poisson probability distribution:

$$
p(x \mid \mu)=e^{-\mu} \frac{\mu^{x}}{x !}
$$

where $\mu$ is a parameter describing the expected number of occurrences.

In this paper, three values of $\mu$ have been assessed for each case study listed in Table $2,0.0,0.5$ and 1.0. Note that for $\mu=0.0$ only ownship wind observations are used to update $c$, since $p(x \mid 0)=0 \forall x \in \mathbb{N}$. In addition, the reference situation in which the initial wind profile forecast obtained from RAP is never updated (i.e., the wind profile is static) yet the descent is executed using NMPC guidance, and the worst-case situation in which, at each $\tau_{i}, i=0,1, \ldots, N-1$, the optimal control $\boldsymbol{u}_{i}^{*}$ from the initial trajectory plan (computed at the TOD) is injected to the system in open-loop (OL), are also assessed.

Algorithm 2 outlines the steps performed to update $c$ at $\tau_{i}$.

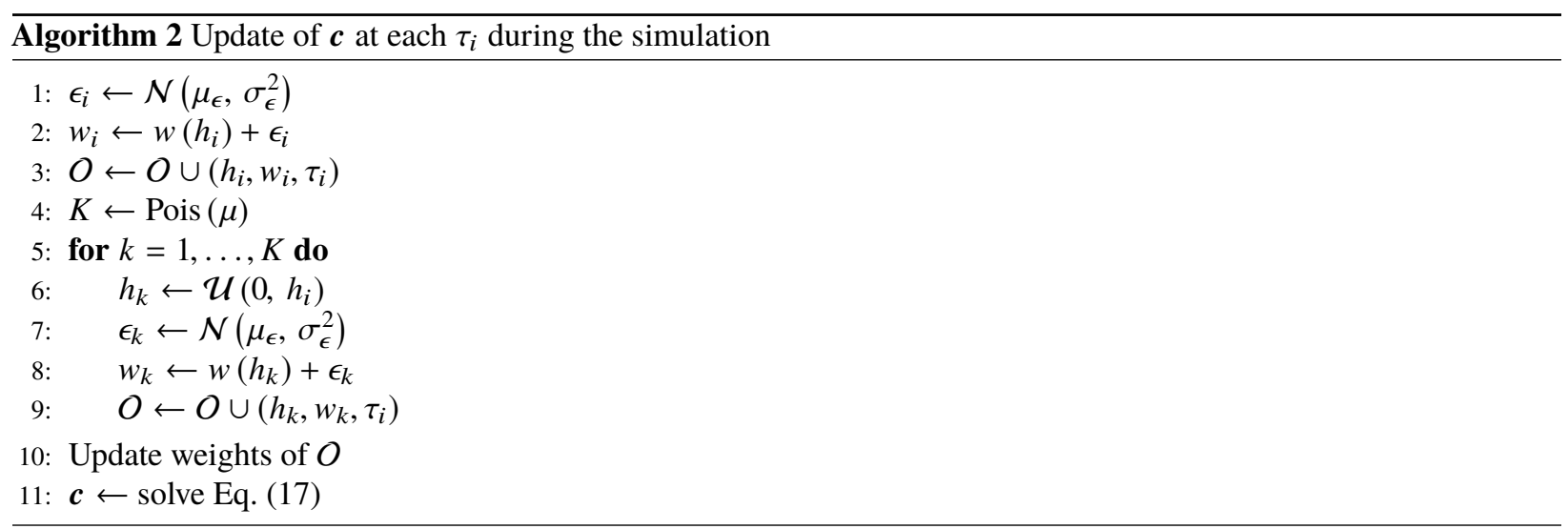

First, the wind sensed by the aircraft at $h_{i}$ was appended to the set of observations. Then, the number of wind observations received from nearby aircraft between $\tau_{i-1}$ and $\tau_{i}, K$, was generated from the Poisson probability distribution. For each wind observation $k=1, \ldots, K$, the altitude $h_{k}$ at which an hypothetical nearby aircraft sensed the wind was generated from a uniform distribution in the altitude interval $\left[0, h_{i}\right]$. The sensed wind included a measurement error $\epsilon_{k}$ generated from a normal distribution, which was centered at $\mu_{\epsilon}=0$ with a standard deviation of $\sigma_{\epsilon}=1 \mathrm{kt}$. The different wind observations $\left(h_{k}, w_{k}, \tau_{i}\right) k=1, \ldots, K$ were progressively appended to $O$. Finally, the weights of the wind observations included in $O$ were updated and $c$ was re-calculated. 
After updating $c$, the optimal trajectory starting at the current state was re-calculated by solving $\mathcal{P}_{N-i}$ according to a more accurate prediction of the actual wind conditions.

In this paper, $\mathcal{P}_{N-i}$ was formulated in CasADi [35], a symbolic framework for automatic differentiation and numeric non-linear optimization, and solved by using the sequential quadratic programming (SQP) algorithm implemented by SNOPT (Sparse Non-linear OPTimizer) NLP solver.

Last but not least, it should be noted that, in practise, the pilot is the responsible to deploy speed-brakes when alerted by the guidance system. In addition, available speed-brakes actions are typically discrete. Consequently, the experiment of this paper was performed under two important assumptions regarding the speed-brakes use: zero delay and perfect execution from the hypothetical pilot, right after being alerted by the guidance system; and a continuous, fine-grained, adjustment of the speed-brakes angle of deflection.

\section{Results}

Section V.A describes a particular case study for various values of $\mu$, as illustrative example. The aggregated results for all case studies and values of $\mu$ are discussed in Section V.B.

\section{A. Illustrative example}

Case study 09 is the illustrative example. Figure 2 shows the planned and executed trajectories, where each panel corresponds to a different rate of wind observations available to update the wind profile. The lightest solid lines in the three panels of Fig. 2 are identical to each other, i.e., the initially planned trajectory (computed at the TOD). Then, the slightly darker solid lines in Fig. 2 represent the plans resulting from trajectory updates at two of the sixty time samples. These time samples, which were selected only for illustrative purposes and without any specific criteria, are $\tau_{10}$ and $\tau_{40}$. Data for the remaining time samples are not shown for the sake of clarity.

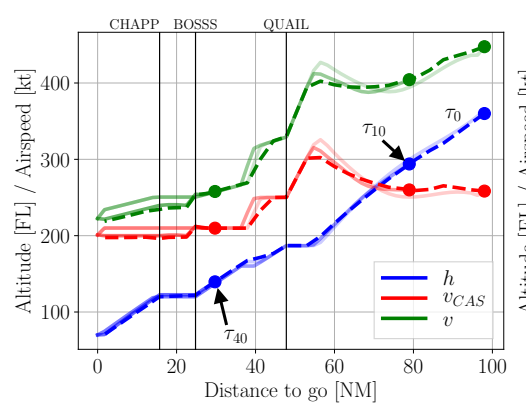

(a) Only ownship observations $(\mu=0.0)$

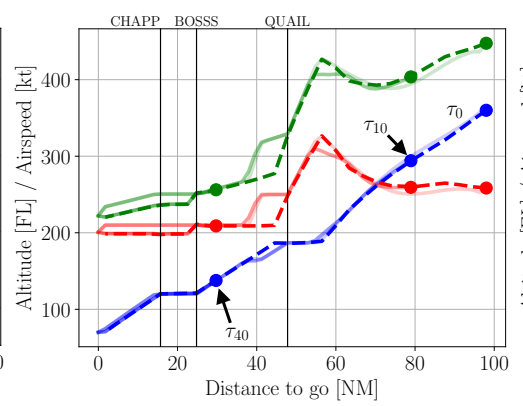

(b) $\mu=0.5$

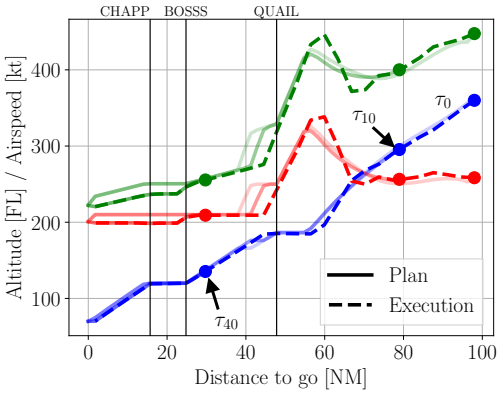

(c) $\mu=1.0$

Fig. 2 Planned and executed trajectories by $\mu$ (case study 09)

Before updating the trajectory at each time sample, the NMPC guidance system updates the wind profile according to Algorithm 2 if networked wind data are available. If this were the case, the forecast wind profile would converge to 


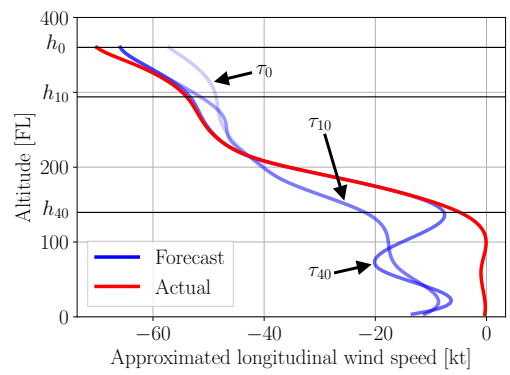

(a) Only ownship observations $(\mu=0.0)$

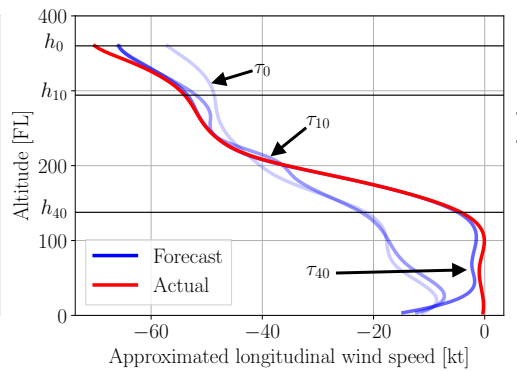

(b) $\mu=0.5$

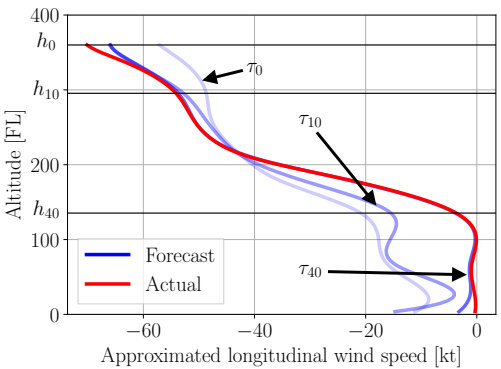

(c) $\mu=1.0$

Fig. 3 RAP wind forecast and analysis by $\mu$ (case study 09)

the actual wind profile. When networked wind data are not available, the forecast wind profile remains static and does not converge to the actual wind profile. This can be observed in Fig. 3 , which shows the initial wind forecast, the wind forecast at the time samples $\tau_{10}$ and $\tau_{40}$, and the actual wind profile.

In Fig. 3 , the forecast wind profile (lightest blue line) of case study 09 deviated fairly substantially from the actual wind (red line) in two different portions of the CDO. From FL360 to FL220 the forecast wind profile underestimated the actual headwind (negative values of X-axis), and from FL200 to the surface it overestimated the actual headwind. Therefore, when analyzing the error in the forecast wind profile shown in Fig. 3 , the expectation would be an aircraft would have to increase its airspeed from FL360 to FL220 and decrease its airspeed below FL200 from the initial calculated trajectory. A closer examination of Fig. 2] reveals this does in fact happen. In Fig. 2(b) for $\mu=0.5$, the speed of the aircraft increases around 15 NM before QUAIL and decreases afterwards, if compared to the initial plan, in order to satisfy the operational constraints enforced at the waypoints of the route. In Fig. 2(c) for $\mu=1.0$, the speed up is performed much earlier (around 25 NM before QUAIL) since a better knowledge of the actual wind profile downstream is available well in advance (see Figs. 3(b) and 3(c), respectively). In all cases, after QUAIL the updated trajectory requires the aircraft to slow its calibrated airspeed to compensate for the weaker than expected head wind from the initial forecast.

Fig. 3(a) shows that when only own measurements are used to update the wind forecast, any error in the forecast will not be corrected at downstream waypoints. On the other hand, when wind observations from other aircraft are considered in the estimation of the wind profile, up-to-date wind data in the whole range of altitudes may be accessible. As expected, the wind forecast converges earlier to the actual wind profile as the rate of wind observations received per time interval increases.

For this particular case study, the time error resulting from executing the optimal control of the initial plan in open-loop was $76 \mathrm{~s}$. When implementing the NMPC guidance strategy, independently of the mechanism selected to manage the wind profile forecast, the time error was reduced to values below $30 \mathrm{~s}$. Using a static wind profile, the time error was $27 \mathrm{~s}$. The smallest time error (10 s) was achieved for $\mu=1.0$. 
In terms of pseudo-specific energy error at the metering fix, the deviation with respect to the initial plan for the open-loop execution was around $1250 \mathrm{ft}$. For the NMPC strategy the $E_{s}$ error was negligible, being lower than $50 \mathrm{ft}$ independently of the mechanism selected to manage the wind profile forecast. Again, the best case was achieved with $\mu=1.0$.

When a fully wind networked concept concept is implemented (Fig. 2(c) with $\mu=1.0$ ), the executed trajectory is changed sooner and more profoundly than when less or no updated wind information is available as shown by the larger difference between the light and dark red lines.

\section{B. Aggregated results}

This section presents the performance metrics for all case studies of the experiment. Fig. (4) a) shows the time error at the metering fix (DYMON) with respect to the enforced CTA for three different values of $\mu$ and for the case in which the wind forecast was not updated, i.e., the initial wind forecast from RAP was kept static throughout the descent. The time error that would be achieved by applying the optimal control resulting from the initial plan in open-loop is also shown. Analogously, Fig. 4 (b) shows the $E_{s}$ error at DYMON.

According to Fig. 4(a), the time error at the metering fix when applying the optimal control from the initial plan in open-loop could be higher than $100 \mathrm{~s}$. When using NMPC guidance, the time error was drastically reduced and typically less than $15 \mathrm{~s}$. For the static wind profile approach, in which the trajectory is updated at each time sample based on a wind forecast that does not change during the execution of the descent, the time error was lower than $30 \mathrm{~s}$ for all case studies. The larger time error corresponds to case study 08 , in which the time error was similar to that of the open-loop execution. For the simulations in which the wind forecast was updated with only ownship observations, the time error was lower than $17 \mathrm{~s}$ for all case studies. Using this approach, the time error was approximately halved with respect to that using a static wind forecast. Results show that if additional wind data emitted by aircraft in range were used to update the wind profile, the time error would be negligible for all case studies.

According to Fig. $4\left(\right.$ b), the metering fix could be achieved with $E_{s}$ errors up to $1600 \mathrm{ft}$ by implementing the optimal control of the initial plan in open-loop. Conversely, when executing the descent with NMPC the metering fix is achieved with much smaller error. For the static wind profile approach, the $E_{s}$ error is lower than $200 \mathrm{ft}$ for all case studies. When the wind profile is updated at each time sample yet using only ownship wind observations, the maximum $E_{S}$ error is reduced to $50 \mathrm{ft}$. Finally, if additional wind observations from nearby aircraft were used to update the wind profile at each time sample, the $E_{s}$ error would be negligible for all case studies.

Either a deficit of energy (low and/or slow) or an excess of energy (high/fast) at the metering fix may result in an incident or accident. Typical incident/accidents due to an incorrect energy management during the descent are hard landing, trail strike, and runway overrun or undershoot. The acceptable pseudo-specific energy error at the metering fix depends on many factors, including the remaining distance to go, the state of the aircraft (altitude and speed) and its 

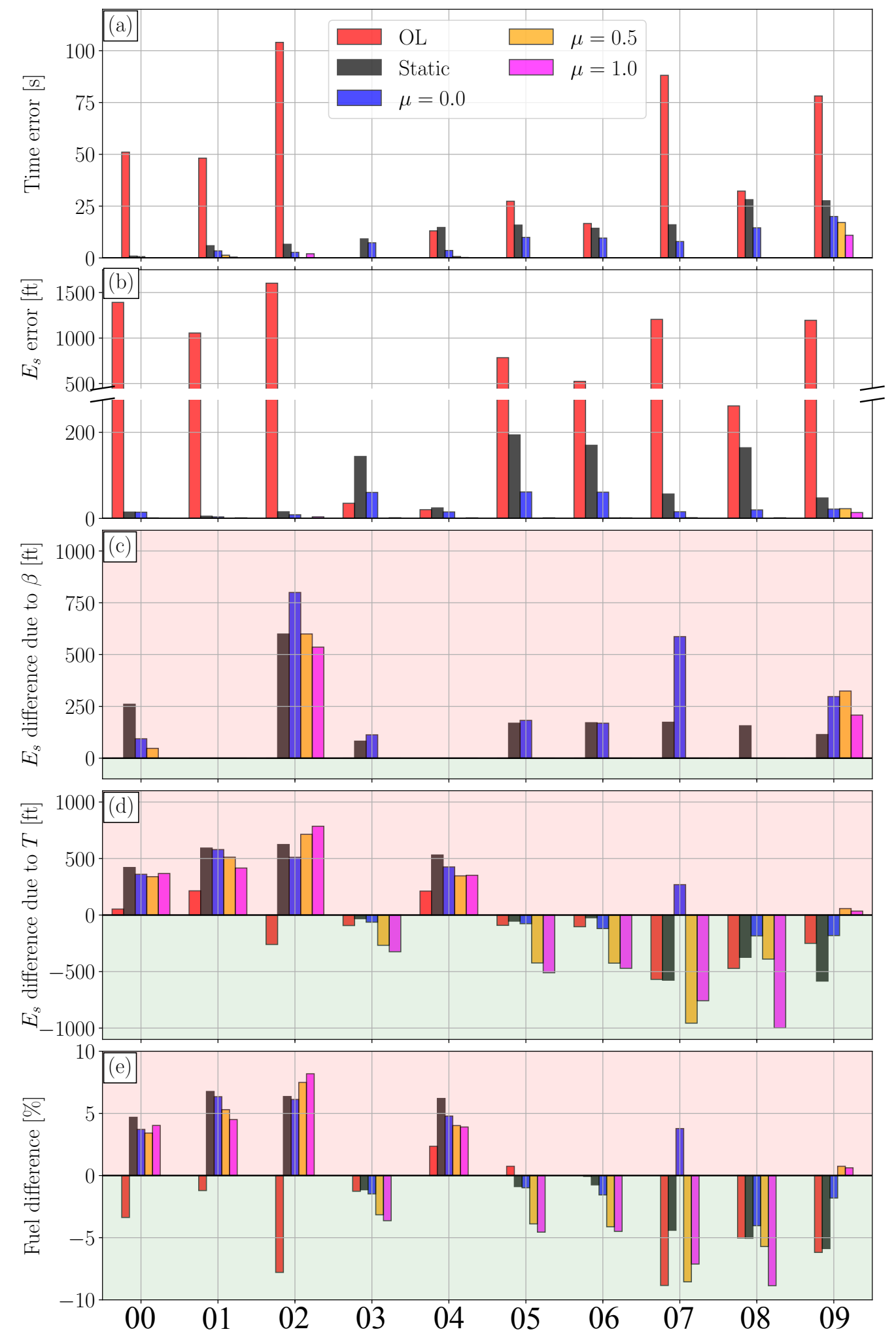

Fig. 4 Aggregated results at metering fix by case study 
mass, the amount of energy that the aircraft can still add/remove by means of thrust/speed-brakes use (i.e., how far it is from the control limits), as well as the weather conditions, being the longitudinal wind one of the key factors.

In the methodology proposed in this paper, the optimal control computed at each $\tau_{i}$ modulates the energy of the aircraft by using the elevator to change the aircraft's airspeed to meet constraints and achieve the CTA. When this is insufficient in certain wind conditions, it then increases thrust (adds energy) or deploys speed brakes (removes energy). In this case, the NMPC trajectory optimizer would calculate the optimal amount of energy to be added or removed in terms of fuel consumption and speed brakes use such that all constraints are satisfied.

Fig. 4(c) shows, for each case study, the difference between the executed trajectory and the initial plan, in terms of total pseudo-specific energy removed by deploying the speed brakes. Positive values indicate that trajectory updates removed more energy by using speed brakes than initially planned. Fig. 4 (d) shows the difference in pseudo-specific energy added by thrust between the initial plan and executed plan, with positive values indicating more thrust was used than initially planned.

According to Fig. 4(c), the static wind profile approach requires speed brakes in 8 of the 10 case studies assessed herein. When the wind profile is updated with only ownship wind observations, similar energy needs to be removed by deploying speed brakes, but on the other hand the time error and the pseudo-specific energy errors at the metering fix are appreciably smaller. A significant reduction of the pseudo-specific energy removed by deploying speed brakes is observed when including wind observations of nearby aircraft in the prediction of the wind profile. In particular, for $\mu=0.5$ only 3 of 10 case studies required the use of speed brakes, and only 2 case studies for $\mu=1.0$. It should be noted that, for this particular set of case studies, incorporating more wind observations does not only reduce the number of case studies where speed brakes were required, but also the total amount of energy removed.

The planned thrust is based on the aircraft's altitude and airspeed along the arrival procedure. When the aircraft deviates from the planned altitude and/or airspeed, the thrust actually used may be different from that of the initial plan at the same $\tau_{i}$. Therefore, the planned thrust at $\tau_{i}$ could be slightly different from the actual thrust at $\tau_{i}$. These state deviations are the cause of the pseudo-specific energy differences shown in Fig. 4.d) for the OL strategy.

According to Figs. 4(c) and 4(d), the results from the static wind forecast required the use of speed brakes and throttles compared to other guidance strategies. For $\mu=0.0$, only one energy-neutral descent (i.e., requiring neither additional thrust nor speed brakes during the descent) was obtained, corresponding to case study 08 . For $\mu=0.5$ and $\mu=1.0$, the number of energy-neutral descents greatly increased to 5 . These are very promising results considering that the case studies selected for this experiment correspond to the worst RAP forecasts generated for +3 and +6 hours look-ahead times during one year. It should be noted that for those case studies in which energy modulation was not sufficient to satisfy operational constraints, the amount of energy added/removed by the NMPC guidance system was optimal in terms of fuel consumption and use of speed brakes.

Fig. 4(e) shows the difference in fuel consumption between the executed trajectory and the initial plan, with negative 
values indicating fuel savings and positive values indicating that additional fuel was required. Results shown in Fig.4.e) agree with those of Fig.4(d): the cases studies that required additional pseudo-specific energy (i.e., thrust), typically resulted in extra fuel consumption. Interestingly, compared to the initial trajectory plan, the extra fuel required by the NMPC to compensate energy and time deviations when considering the worst wind forecast during one year at Denver is less than $10 \%$. Furthermore, for most of the case studies analysed herein, adding wind observations from nearby aircraft resulted in fuel savings if compared to the initial trajectory plan. These results indicate that NMPC combined with wind networking concepts could be a mechanism to correct time and energy deviations at no extra fuel cost (at aggregated level). However, a more deep analysis considering a large number of case studies should be performed to support this hypothesis.

\section{Conclusions}

This paper combined a non-linear model predictive control (NMPC) guidance strategy, which repeatedly updates the optimal trajectory of an aircraft, with a wind networking concept in which aircraft and ground systems share wind observations to improve the wind profile forecast on-board and in real-time.

Results from simulations considering the 10 top worst wind forecast during one year at Denver show that the performance of the NMPC strategy is significantly improved when including up-to-date wind observations, in terms of time and energy errors at the metering fix and fuel consumption. The proposed approach has shown to potentially compensate large energy and time errors using minimum fuel consumption. While this paper focused on the worst-case scenarios, future work should assess also the performance of the proposed algorithm, in terms of for example the number of energy-neutral descents, for different wind forecasts quality.

Unfortunately, the current and accurate ADS-B and Mode-S data being transmitted is not currently being used to update the wind profile used by the FMS to optimize the trajectory plan. Results arising from this work should encourage the aviation community to take advantage of data provided in the wind networked concept to further optimize the trajectory.

Last but not least, the method proposed in this paper requires both ADS-B and Mode-S data to derive accurate estimates of the wind direction and speed. Mode-S messages, however, are only sent in response to secondary surveillance radar interrogation from air traffic control, meaning that this kind of messages may not be always available. The improvement in time predictability and the reduction of environmental impact shown in this paper call out the upcoming changes to the ADS-B standard to incorporate weather information into the ADS-B message.

\section{Acknowledgments}

The authors acknowledge the technical support during the development of the guidance algorithms presented in this paper of Dr. Joel Andersson, one of the main authors of the CasADi software package. Much appreciation goes out to 
Prof.Dr.-Ing. Wolfgang Marquardt and Dr. Ralf Hannemann-Tamás for their expertise in the NMPC field and advice.

\section{References}

[1] Wynnyk, C., Balakrishna, M., MacWilliams, P., and Becher, D. T., “2011 Trajectory Based Operations Flight Trials,” 10th USA/Europe Air Traffic Management R\&D Seminar (ATM2013), Chicago , IL, 2013.

[2] "Minimum aviation system performance standards: required navigation performance for area navigation change 1,", September 2014. Standard: RTCA DO-236.

[3] Klooster, J., Wichman, K., and Bleeker, O., “4D Trajectory and Time-of-Arrival Control to Enable Continuous Descent Arrivals," AIAA Guidance, Navigation and Control Conference and Exhibit, Guidance, Navigation, and Control and Co-located Conferences, Honolulu, Hawaii, 2008. doi:10.2514/6.2008-7402.

[4] Bronsvoort, J., McDonald, P. R., G., and Gutt, E., "Enhanced Descent Wind Forecast for Aircraft: Facilitation of Continuous Descent Arrivals with Improved Efficiency and Predictability by the use of Tailored Descent Wind Forecasts," Proceedings of the 9th USA/Europe Air Traffic Management Research and Development Seminar, Berlin, Germany, 2011.

[5] Green, S. M., Grace, M. P., and Williams, D. H., "Flight Test Results: CTAS and FMS Cruise/Descent Trajectory Prediction Accuracy," Proceedings of the 3rd USA/Europe Air Traffic Management Research and Development Seminar, Napoli, Italy, 2000 .

[6] Prats, X., Dalmau, R., Verhoeven, R., and Bussink, F., "Human-in-the-loop Performance Assessment of Optimized Descents with Time Constraints," Twelfth USA/Europe Air Traffic Management Research and Development Seminar (ATM2017), FAA and EUROCONTROL, Seattle, WA, 2017.

[7] Green, S. M., Vivona, R. A., and Grace, M. P., "Field Evaluation of Descent Advisor Trajectory Prediction Accuracy for En-route Clearance Advisories," AIAA Guidance, Navigation, and Control Conference, 1998. doi:10.2514/6.1998-4479.

[8] Green, S. M., and Vivona, R. A., "Field evaluation of Descent Advisor trajectory prediction accuracy," AIAA Guidance, Navigation, and Control Conference, 1996. doi:10.2514/6.1996-3764.

[9] Oseguera, R. M., and Williams, D. H., "Flight evaluation of the CTAS descent advisor trajectory prediction," Proceedings of 1995 American Control Conference - ACC 95, Vol. 5, 1995, pp. 3435-3439.

[10] Glina, Y., Troxel, S., Reynolds, T., , and McPartland, M., "Wind Information Requirements to Support Four Dimensional Trajectory-Based Operations," Proceedings of the 12th AIAA Aviation Technology, Integration, and Operations (ATIO) Conference and 14th AIAA/ISSM, Indianapolis, Indiana, 2012.

[11] Diehl, M., Ferreau, H. J., and Haverbeke, N., "Efficient Numerical Methods for Nonlinear MPC and Moving Horizon Estimation Problem Formulation,” Workshop on Assessment and Future Directions of NMPC, Pavia, Italy, 2008, pp. 391-417. doi:10.1007/978-3-642-01094-1_32. 
[12] Dalmau, R., Prats, X., Verhoeven, R., Bussink, F., and Heesbeen, B., "Performance comparison of guidance strategies to accomplish RTAs during a CDO,” 36th IEEE/AIAA Digital Avionics Systems Conference (DASC), St. Petersburg, FL, 2017.

[13] Weitz, L. A., and Bai, X., "Using Model Predictive Control for Trajectory Optimization and to Meet Spacing Objectives," AIAA Guidance, Navigation, and Control Conference, Kissimmee, FL, 2018.

[14] Pesch, H. J., "Numerical computation of neighboring optimum feedback control schemes in real-time," Applied Mathematics \& Optimization, Vol. 5, No. 1, 1979, pp. 231-252. doi:10.1007/BF01442556.

[15] Würth, L., Hannemann, R., and Marquardt, W., “An Efficient Strategy for Real-Time Dynamic Optimization based on Parametric Sensitivities," IFAC Proceedings Volumes, Vol. 41, No. 2, 2008, pp. 1928-1933. doi:10.3182/20080706-5-KR-1001.00328.

[16] Dalmau, R., Prats, X., and Baxley, B., "Fast sensitivity-based optimal trajectory updates for descent operations subject to time constraints," 38th IEEE/AIAA Digital Avionics Systems Conference (DASC), London, UK, 2018.

[17] in 't Veld, A., "Self-Spacing Algorithms for Continuous Descent Approaches," Ph.D. thesis, Technische Universiteit Delft, June 2011.

[18] de Jong, P. M. A., van der Laan, J. J., Veld, A. C., van Paassen, M. M., and Mulder, M., "Wind-Profile Estimation Using Airborne Sensors,” Journal of Aircraft, Vol. 51, No. 6, 2014, pp. 1852-1863. doi:10.2514/1.C032550.

[19] Abbott, T. S., and Swieringa, K. S., "An Overview of a Trajectory-Based Solution for En Route and Terminal Area Self-Spacing: Eighth Revision,” Tech. rep., NASA Langley Research Center, Hampton, VA, October 2017.

[20] Bussink, F. J. L., Van der Laan, J. J., and De Jong, P. M. A., "Combining Flight-deck Interval Management with Continuous Descent Approaches in high density traffic and realistic wind conditions," Proceedings of the AIAA Guidance, Navigation and Control Conference, Minneapolis, Minnesota, 2012.

[21] Betts, J. T., Practical Methods for Optimal Control and Estimation Using Nonlinear Programming, $2^{\text {nd }}$ ed., SIAM, 2010. doi:10.1137/1.9780898718577.

[22] Jäschke, J., Yang, X., and Biegler, L. T., "Fast economic model predictive control based on NLP-sensitivities," Journal of Process Control, Vol. 24, No. 8, 2014, pp. 1260-1272. doi:10.1016/j.jprocont.2014.04.009.

[23] Haan, S. D., "Quality assessment of high resolution wind and temperature observation from ModeS,” Tech. rep., The Royal Netherlands Meteorological Institute (KNMI), The Netherlands, 2010.

[24] Bienert, N., and Fricke, H., "Real-timeWind Uplinks for Prediction of the Arrival Time and Optimization of the Descent Profile," Proceedings of the 3rd ENRI International Workshop on ATM/CNS, Tokyo, Japan, 2013.

[25] W.M. Hollister, Bradford, E., and Welch, J. D., "Using aircraft radar tracks to Estimate winds aloft," The Lincoln Laboratory Journal, Vol. 2, No. 3, 1986, pp. 555-565. 
[26] De Leege, A. M. P., Mulder, M., and Van Paassen, M. M., "Using Automatic Dependent Surveillance-Broadcast for Meteorological Monitoring," Journal of Aircraft, Vol. 50, No. 21, 2013, pp. 249-261.

[27] Delahaye, D., and Puechmorel, S., "TAS and wind estimation from radar data," 28th AIAA/IEEE Digital Avionics Systems Conference Proceedings, Orlando, United States, 2009, pp. 2.B.5-1 -2.B.5-16. doi:10.1109/DASC.2009.5347547.

[28] Hrastovec, M., and Solina, F., "Obtaining meteorological data from aircraft with mode-S radars," IEEE Aerospace and Electronic Systems Magazine, Vol. 28, No. 12, 2013, pp. 12-24. doi:10.1109/MAES.2013.6693664.

[29] Dalmau, R., Pérez-Batlle, M., and Prats, X., "Estimation and prediction of weather variables from surveillance data using spatiotemporal Kriging," 37th AIAA/IEEE Digital Avionics Systems Conference (DASC), 2017. doi:10.1109/DASC.2017.8102132.

[30] Sun, J., Vu, H., Ellerbroek, J., and Hoekstra, J., "Ground-based Wind Field Construction from Mode-S and ADS-B Data with a Novel Gas Particle Model," 7th SESAR Innovation Days, Belgrade, Serbia, 2017.

[31] Clarke, J. P. B., Ho, N. T., Ren, L., Brown, J. A., Elmer, K. R., Zou, K. F., Hunting, C., McGregor, D. L., Shivashankara, B. N., Tong, K., Warren, A. W., and Wat, J. K., "Continuous descent approach: Design and flight test for Louisville international airport," Journal of Aircraft, Vol. 41, No. 5, 2004, pp. 1054-1066. doi:10.2514/1.5572.

[32] de Boor, C., "On calculating with B-splines," Journal of Approximation Theory, Vol. 6, No. 1, 1972, pp. 50-62. doi: 10.1016/0021-9045(72)90080-9.

[33] Poles, D., Nuic, A., and Mouillet, V., "Advanced aircraft performance modelling for ATM: Analysis of BADA model capabilities," 29th Digital Avionics Systems Conference, EUROCONTROL, Brétigny-sur-Orge (France), 2010. doi:10.5772/2706.

[34] Airbus, "Getting to grips with the cost index," Tech. rep., Airbus Flight Operations Support \& Line Assistance, Blagnac, France, May 1998.

[35] Andersson, J., Akesson, J., and Diehl, M., "Dynamic optimization with CasADi," Proceedings of the IEEE Conference on Decision and Control, 2012, pp. 681-686. doi:10.1109/CDC.2012.6426534. 